\title{
Une méthode d'évaluation régionale de la biomasse des taillis à partir des données de l'Inventaire forestier national. Application à la région Centre
}

\author{
D. AUCLAIR et Marie-Claude BIGE (*) \\ I.N.R.A., Station de Sylviculture \\ Centre de Recherches forestières d'Orléans, Ardon, F 45160 Otivet
}

\begin{abstract}
Résumé
Les publications de l'Inventaire forestier national fournissent des renseignements sur lc volume de bois fort (VBF) des peuplements forestiers, selon les différentes régions, essences, âges, etc. Le présent travail propose une méthode permettant de convertir ces données en biomasse sèche totale aérienne (BST). Une régression du type $B S T=a \mathrm{VBF}+\mathrm{b}$ a donné de bons résultats : $\mathrm{r}^{2}=0,961$ sur 142 individus provenant de 96 parcelles de tállis ou taillis sous futaie réparties en région Centre. Si certaines des variables étudiées ont une influence sur les coefficients de la régression (en particulier la classe d'âge 0 à 9 ans comprenant de très petits brins), l'équation globale est très satisfaisante avec un écart-type de 1,8 p. 100 sur le brin moyen. L'étude des petits brins n'ayant pas de bois fort et l'emploi de cette équation ont permis de donner une estimation globale de la biomasse sur pied des peuplements de la région Centre. On peut noter que la contribution de brins sans bois fort n'est que de 12 p. 100 sur les parcelles échantillonnées. L'extrapolation à la France entière telle qu'elle est suggérée ici ne peut être que provisoire, et nécessite une ćtude plus poussée avec un échantillonnage plus complet couvrant toutes les régions.
\end{abstract}

\section{Introduction}

\subsection{L'Inventaire forestier national}

La Direction des Forêts du ministère de l'Agriculture possède un service chargé de l'Inventaire forestier national (I.F.N.). La connaissance des ressources est en effet une donnée de base pour l'aménagement de l'espace forestier, et la prévision des ressources disponibles dans l'avenir constitue une donnée nécessaire pour fonder une politique forestière.

(*) Travail effectué pendant le stage de fin d'études de M. C. Bige (E.N.S.F.A., Remes) avec laide du Service Technique Biomasse et de MM. Bedeneau, Corbon, I Timint, Mortal et ROMARY. 
Unc première couverture de toute la surface du pays a été effectuée département par département, et une remise à jour est actuellement en cours. Des renseignements sont donnés sur les forêts, les formations boisées dans le domaine agricole, et les landes. Sont ainsi évalués les surfaces, les volumes sur pied, les accroisscments annuels de ces volumes, et le nombre d'arbres, en fonction des grandes catégories de propriétés, types de forêts, traitements, essences, classes d'âge, catégories de dimensions, densités.

La méthode d'inventairc, décrite en détail dans la publication I.F.N. (1972) : "But et méthodes de l'Inventaire forestier national ", consiste en 3 phases : une

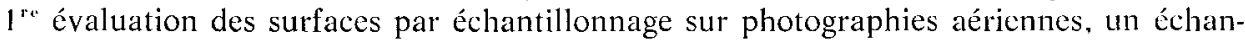
tillonnage de contrôle au sol de ces surfaces, et un échantillonnage au sol détaillé en vue de mesurer les différentes caractéristiques des peuplements.

Les résultats sont actuellement présentés dans des tableaux répartis pour chaque département en 2 tomes : le $1^{\prime r}$ réunit les résultats globaux de surfaces, volumes et accroissements, tant pour les forêts que pour les formations boisées, et fournit une analyse des données recueillies. Le $2^{\prime \prime}$ tome réunit des résultats plus détaillés au niveau des essences et des types de peuplement des seules formations boisées de production.

Les volumes mesurés sont définis de la manière suivante :

- volume sur écorce arrêté à la découpe "bois fort » $(7 \mathrm{~cm}$ de diamètre) pour la tige des résineux et des peupliers et celle des feuillus appartenant aux catégories des bois moyens et des petits bois, y compris les brins de taillis;

- volume sur écorce arrêté à la "découpe marchande» de $20 \mathrm{~cm}$ pour les tiges des feuillus appartenant à la catégorie gros bois (diamètre à $1,30 \mathrm{~m}$ supérieur à $37.5 \mathrm{~cm}$ ).

\subsection{La biomasse}

Les connaissances fondamentales sur le fonctionnement des écosystèmes nécessitent l'évaluation de la biomasse totale de leurs différents constituants. En outre. avec les perspectives actuclles de pénurie d'énergie et de matières premières, l'utilisation de la forêt se diversifie de façon intéressante. Les produits du bois peuvent être employés sous diverses formes : bois, particules, pâtes, fibres, produits chimiques, énergie.

Les études sur la biomasse des arbres forestiers sont nombreuses et les méthodes d'évaluation de cette biomasse ont déjà été expérimentées et discutées par de nombreux chercheurs (Madgwick, 1976; PArdé, 1980 a; Y'OUnG, 1976 ; Young, 1981). La plupart de ces études ont été effectuées sur des arbres de franc pied, ou arbres de futaie. Peu d'études ont eu lieu sur les taillis, pourtant il existe encore à l'heure actuelle en France environ 2,5 millions d'hectares de taillis simple et 3,7 millions d'hectares de taillis sous futaie (PARdé, $1980 \mathrm{~b}$ ). Citons les études de Auclair \& Metayer (1980) dans la région Centre et de Ranger et al. (1981) dans les Ardennes, donnant des méthodes et des résultats sur quelques pareelles de taillis. 


\subsection{Evaluation régionale}

Pour une bonne gestion de la ressource forestière, que ce soit dans une optique traditionnelle ou pour une utilisation plus moderne de la matière première, il importe de bien connaître l'importance de cette ressource en biomasse totale, et non plus seulement en volume de bois fort. Bouchon et al. (1981) ont effectué une $1^{\text {rn }}$ estimation de la ressource forestière en biomasse totale, en se basant en ce qui concerne les taillis sur nos $1^{\text {er }}$ résultats recueillis sur 3 parcelles en région Centre (Auclair \& Metayer, 1980).

Le but de la présente étude est de donner les moyens d'affiner cette $1^{\text {re }}$ évaluation, à partir de données recueillies sur 96 parcelles de taillis réparties dans la région Centre.

Notons tout d'abord que nous nous sommes restreints au taillis (taillis simple et partie taillis des taillis-sous-futaie). En effet, ce type de peuplement est particulièrement intéressant en ce qui concerne une utilisation indifférenciée de la biomasse forestière. Les arbres de futaie ou les réserves de taillis-sous-futaie sont plutôt destinés à produire du bois de qualité, bien que les déchets ou les rémanents soient également intéressants à prendre en compte. Ceci est étudié par ailleurs par CABANETTES \& LEGUAY (non encore publié).

Notons également que cette étude a été effectuée dans la région Centre. Les résultats sont donc applicables dans cette région, et une extrapolation à la France entière ne doit se faire qu'avec beaucoup de réserves et nécessite en toute rigueur une étude plus poussée dans d'autres régions.

\section{Méthode}

\subsection{Principe}

L'Inventaire forestier national (I.F.N.) fournit des données sur le volume de bois fort. Il s'agit donc ici de trouver des formules permettant de convertir ces données en biomasse totale, ou en biomasse à différentes découpes (1). Unc étude similaire a été effectuée par AlEmDag (1982) sur des peuplements équiennes naturels de Pinus banksiana.

Nous nous sommes basés pour la présente étude sur un échantillonnage de 96 parcelles de taillis ou taillis-sous-futaie, réparties en région Centre, dans différentes conditions. Sur les brins échantillonnés nous avons mesuré le volume de bois fort, ainsi que la biomasse sèche à différentes découpes : bois fort (diamètre $7 \mathrm{~cm}$ ); diamètre $4 \mathrm{~cm}$; diamètre $2,5 \mathrm{~cm}$; biomasse totale aérienne (comprenant les petites branches et les feuilles). Les résultats donnés ici ne concernont que la biomasse totale.

(1) Nous noterons par la suite VBF : volume de bois fort, exprimé en cm" et BST : biomasse sèche totale aérienne, exprimée en grammes. 
Nous avons ensuite cherché une formule simple permettant de prédire la biomasse totale d'un brin de taillis à partir de son volume de bois fort, et l'effet des différentes variables étudićes sur cette formule. Puis nous avons étudié le moyen d'étendre cette formule au niveau du peuplement et des donnécs publiées par l'T.F.N. Ceci pose en particulier un problème dans le cas des petits brins de diamètre à $1,30 \mathrm{~m}$ inférieur à $7 \mathrm{~cm}$. Ceux-ci ont en effet un volume bois fort nul, mais une biomasse totale non nulle.

\subsection{Variables étudiées}

L'I.F.N. fournit des données en fonction de différentes « variables de peuplement $*$. Nous avons étudié l'influence de ces variables sur la relation liant VBF à BST. Il s'agit :

- du type de peuplement : taillis simple, taillis-sous-futaie avec un couvert de réserves inférieur à 50 p. 100 , taillis-sous-futaie avec un couvert de réserves supérieur à 50 p. 100 ;

- de l'essence : chêne, charme et hêtre, bouleau et tremble, châtaignier, autres feuillus. Notons que dans notre étude nous avons regroupé les différentes espèces de chêne : rouvre, pédonculé, pubescent. D'autre part le hêtre est très peu représenté et n'a pas été étudié ici. La catégorie «autres feuillus» a seulement été représentée par le robinier, les autres essences étant trop rares pour justifier un échantillonnage ;

- de l'âge : une sćparation en classes d'âges est effectuée : 0 à 9 ans, 10 à 19 ans, 20 à 29 ans, 30 à 39 ans, supérieur à 40 ans ;

- de la région forestière : 5 régions forestières ont été prospectées : Sologne, Orléanais, Pays Fort. Champagne berrichonne. Boischaut Nord.

Nous avons en outre tenu compte d'une variable qui n'apparaît pas dans les résultats de l'I.F.N., mais qui joue sur le niveau de production :

- le type de sol : podzol, podzolique, lessivé non hydromorphe, lessivé hydromorphe, brun non hydromorphe, brun hydromorphe, hydromorphe (hydromorphic à moins de $35 \mathrm{~cm}$ de profondeur).

\subsection{Travail de terrain}

Dans des peuplements homogènes (les variables précédentes étant constantes) nous avons installé des placettes circulaires de 1 à 3 ares selon l'âge du taillis : classe d'âge 1, 1 are ; classes d'âge 2 à 4,2 ares; classe d'âge 5,3 ares. Le centre de la placette est déterminé au hasard à l'intérieur du peuplement, et son périmètre est délimité grâce à la "mire de Pardé » (PARDÉ, 1961).

Dans cette placette sont choisis au hasard 3 brins sur la cépéc la plus proche du centre ou les cépées voisines, si plus d'une est nécessaire. Les conditions d'environnement sont notées : outre les 5 variables précédentes, on note des données précises sur la densité, le couvert des réserves, les essences secondaires présentes, etc. Le protocole précis d’installation des placettes est décrit dans l'étude de Bige (1982). 
Des mesures de circonférences, biomasses, et volumes sont ensuite effectuées selon la méthode décrite précédemment par Auclair \& Metayer (1980).

Il nous a fallu cependant effectuer une correction, car le volume a été cubé sur le terrain par catégories de diamètre et par la formule « de Smalian ». Cette méthode surévalue le volume, et nous avons calculé un volume «VBF» en assimilant la partie de l'arbre située au-dessus de $1,30 \mathrm{~m}$ et de diamètre supérieur à $7 \mathrm{~cm}$ à une parabole. Nous avons vérifié que le résultat était très proche de celui obtenu en l'assimilant à un cône. Le volume ainsi calculé est équivalent à celui mesuré par l'I.F.N.

\subsection{Analyse des données}

Des régressions linéaires de BST en fonction de VBF ont été calculées pour chaque brin échantillon. Nous avons supprimé dans cette analyse les brins pour lesquels $\mathrm{VBF}=0$.

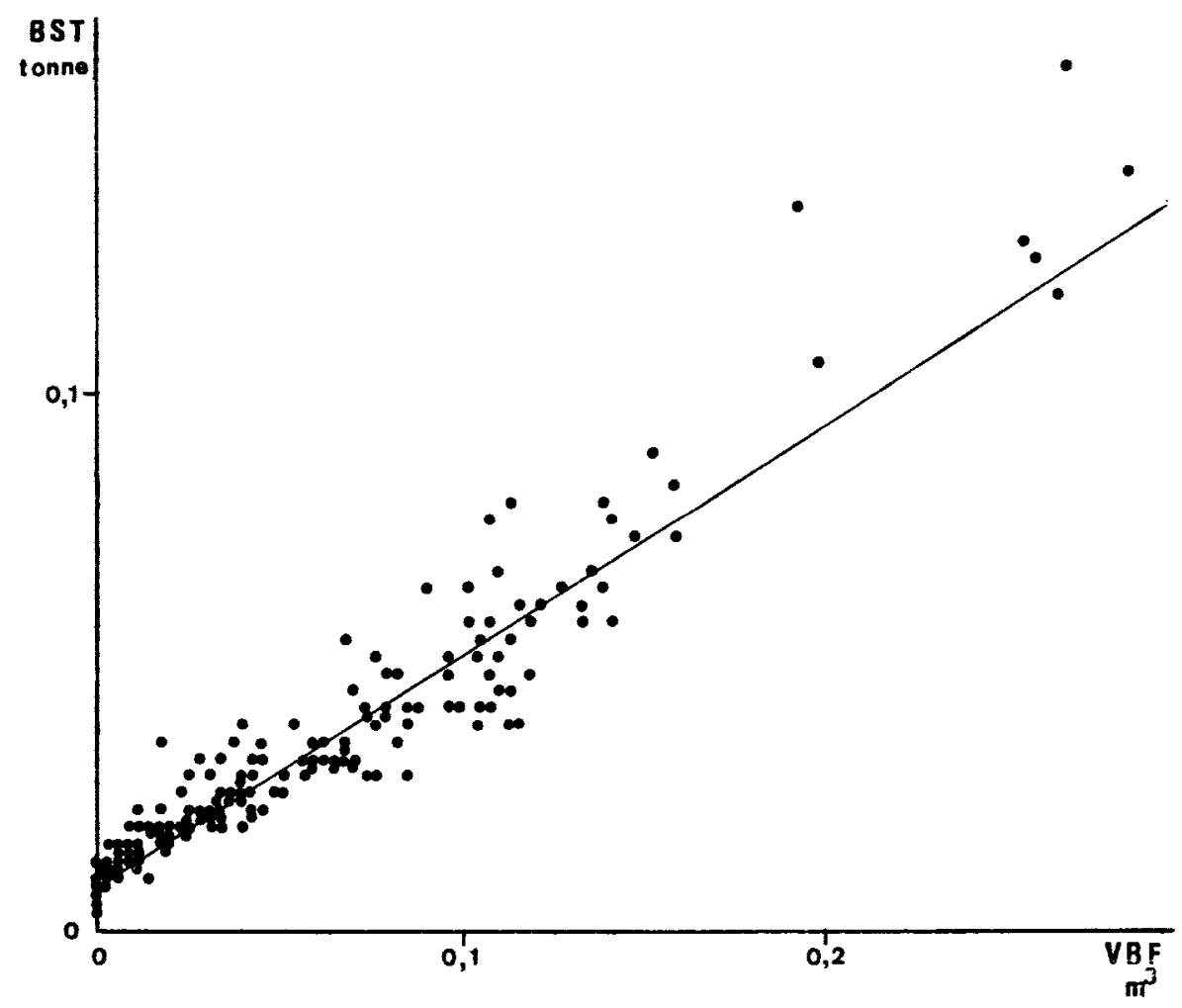

FIG. 1

Représentation graphique de l'ensemble des couples (VBF, BST) et de la droite de régression globale : $B S T(\mathrm{~g})=0,569 \mathrm{VBF}\left(\mathrm{cm}^{\mathrm{s}}\right)+7898$.

Graph showing the distribution of $V B F$ and $B S T$ and the global regression line : BST $(\mathrm{g})=0.569 \mathrm{VBF}\left(\mathrm{cm}^{3}\right)+7898$. 
Nous avons calculé d'une part une régression globale pour les 142 individus ayant $\mathrm{VBF} \neq 0$, d'autre part des régressions partielles pour chacune des valeurs prises par les 5 variables étudiées.

Notons que des régressions de forme plus complexe, logarithmique ou parabolique, ont été testées mais rejetées : la figure 1 montre la bonne linéarité du nuage de points représentant les couples (VBF, BST).

Afin de pouvoir comparer entre elles les équations de régression obtenues nous avons effectué un test d'homogénéité des variances (test de Bartlett, décrit par SNEDECOR \& Cochran, 1971). Nous avons ensuite comparé les régressions par le test décrit par KozaK (1972). Nous avons accepté les hypothèses d'homogénéité des variances, ainsi que de coïncidence ou de parallélisme des régressions, au seuil de 5 p. 100 (test de $\chi^{2}$, test de F).

\section{Résultats et discussion}

\subsection{Régressions par variable (voir note 1)}

\subsection{Régression globale, toutes variables confondues}

Une régression globale a été calculée pour tous les individus ayant du bois fort (fig. 1) :

$$
\mathrm{BST}=0,569 \mathrm{VBF}+7898 \text { pour } 142 \text { individus, } \mathrm{r}^{2}=0,961
$$

\subsection{Type de peuplement:}

(1) taillis simple, (2) taillis-sous-futaie claire, (3) taillis-sous-futaie dense

Les 3 régressions sont comparables d'après le test de Bartlett. Le test de comparaison des régressions montre que les 3 équations coïncident, il est donc inutile de tenir compte du traitement en taillis ou en taillis-sous-futaie, l'équation globale (1) est suffisante (fig. 2).

\subsection{Essence :}

(1) chêne, (2) charme-hêtre, (3) bouleau-tremble, (4) châtaignier,

(5) autres feuillus

Le test de Bartlett permet de comparer les essences 1,3 et 4 , le test de comparaison des régressions permet d'accepter le parallélisme mais non la coïncidence de ces 3 régressions.

En outre, le coefficient de régression de l'essence 5 se trouvant compris entre ceux des essences 3 et 4 , nous pouvons considérer que seule l'essence 2 (charme) se trouve dans une catégorie distincte (fig. 3). 


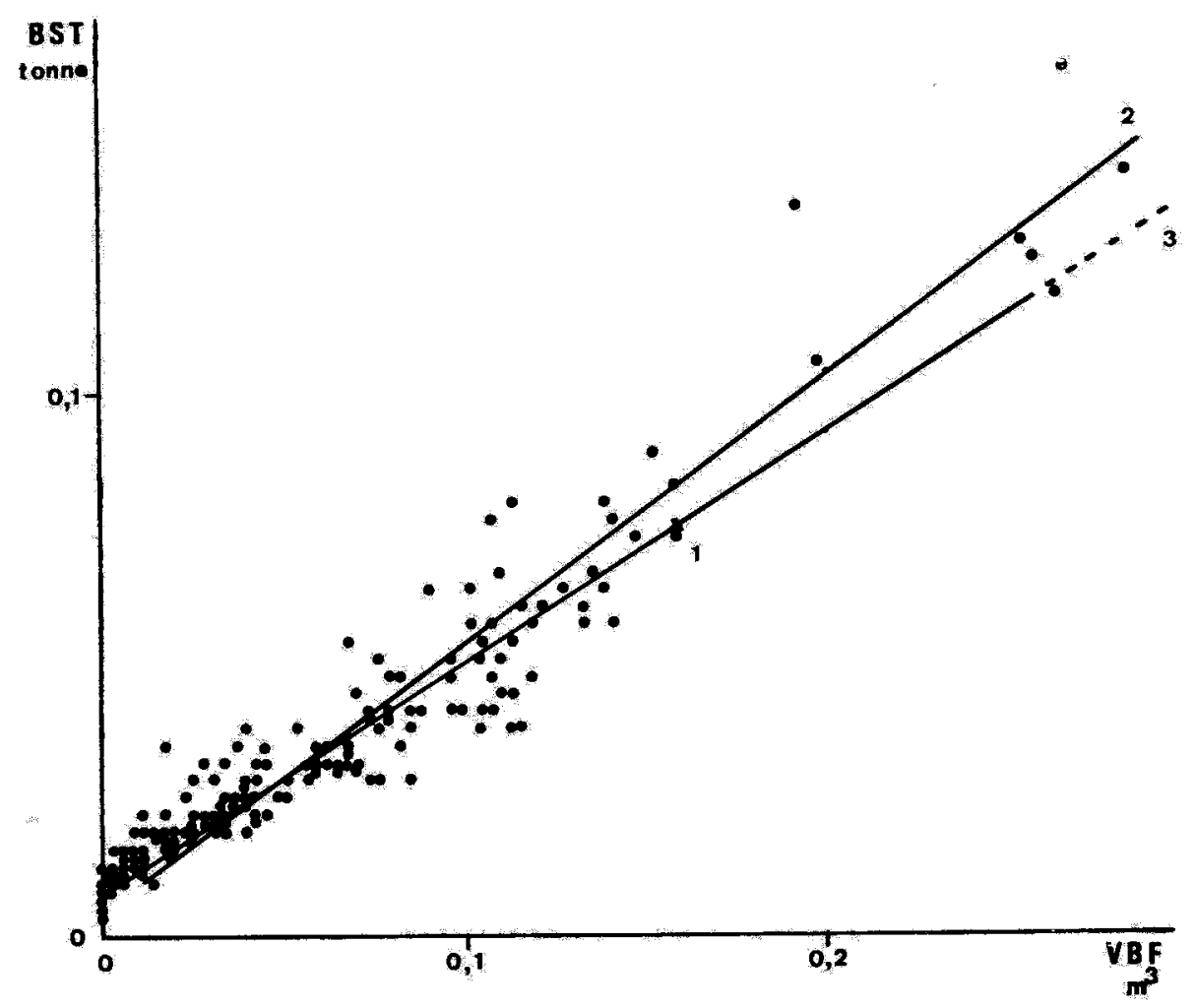

FiG. 2

Représentation graphique des droites de régression pour la variable «Peuplement ».

Graph showing the 3 regression tines for the variable \&stand structure ${ }_{\text {. }}$

1. Taillis simple.

Simple coppice.

2. Taillis sous futaie à couvert inferieur â 50 p. 100 .

Coppice with standards with less than 50 p. 100 standards.

3. Taullis sous futaie à couvert supérieur à $50 \mathrm{p}, 100$.

Coppice with standards with more than 50 p. 100 standards.

Les droites sont représentées en trait plein à l'intérieur de leurs limites de validitế, en pointillếs à textérieur. 


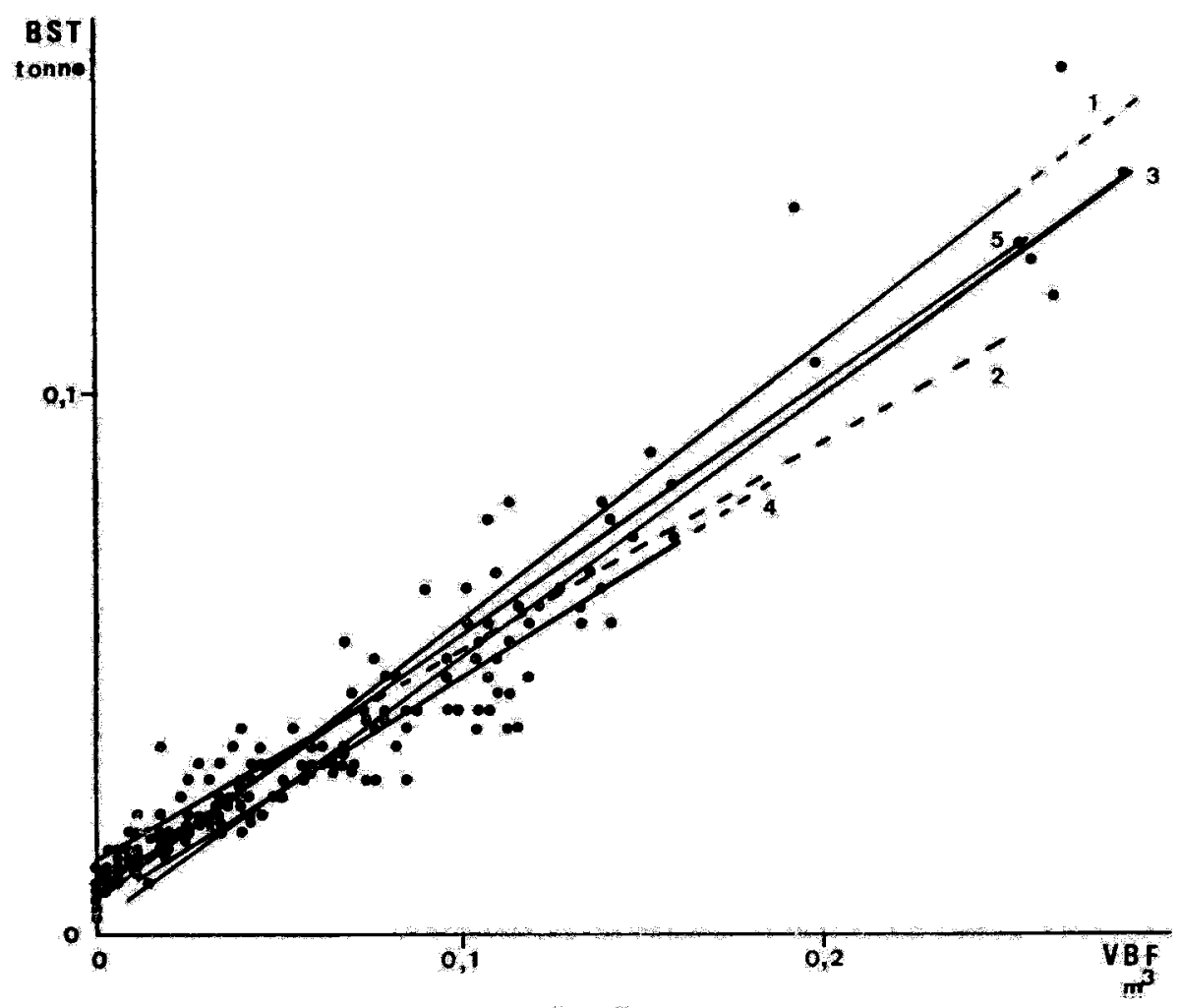

FiG. 3

Représentation graphique des droits de régression pour la variable essence».

Graph showing the 5 regression lines for the variable «species».

1. Chềne:

oak.

2. Charme-hêtre.

Hornbeam-beech.

3. Bouleau-tremble.

Birch-aspen,

4. Châtuaignier.

Chestnut.

5. Autres feuillus (robiníer).

Other (black loctust). 


\subsection{Classe d'âge :}

(1) 0-9 ans, (2) 10-19 ans, (3) $20-29$ ans, (4) 30-39 ans, (5) $>40$ ans

Les 3 classes d'âge 2, 4 et 5 sont comparables (test de Bartlett) et leurs populations peuvent être considérées comme confondues avec une seule équation. La classe 1 qui comprend les très jeunes brins, d'âge inférieur à 9 ans, est peu significative. En effet, ces petits brins n'ayant en général pas de bois fort, seuls 3 brins sont pris en compte dans l'analyse. L'équation de régression ainsi calculée ne doit donc pas être considéréc comme représentative.

Nous pouvons cependant remarquer (fig. 4) que les 3 points considérés se trouvent regroupés dans la partie inférieure gauche du nuage et ne se distinguent pas des autres points du graphique.

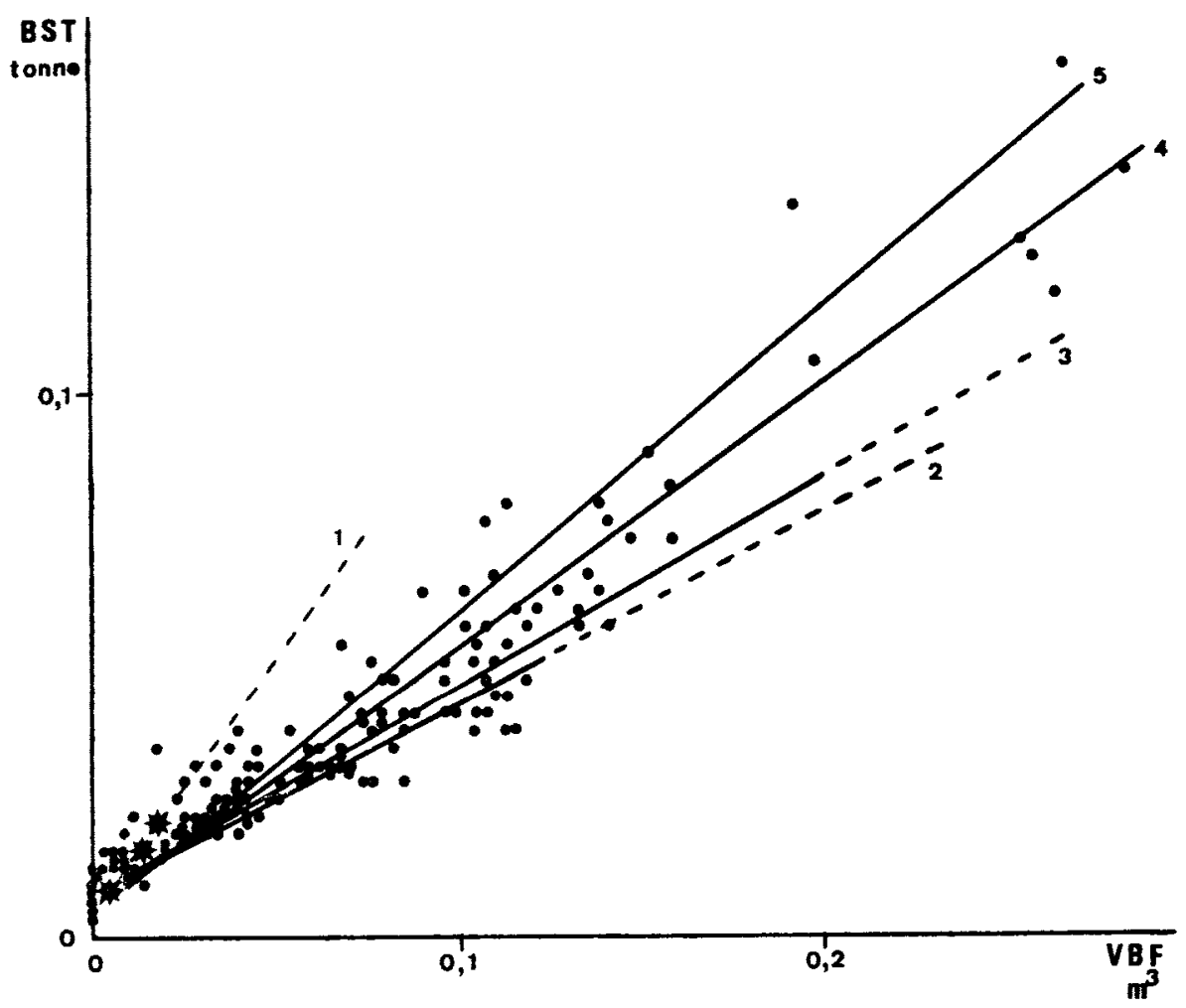

FIG. 4

Représentation graphique des droites de régression pour la variable «Age».

Graph showing the 5 regression lines for the variable «age class».
1. 0 à 9 ans.
$0-9$ years.
2. 10 à 19 ans.
10-19 years.
3.20 à 29 ans.
$20-29$ years.

4. 30 à 39 ans. 30-39 years.
5. Supérieur à 40 ans. Above 40 years.

Les 3 points de la classe 1 ont été représentés par $*$, les points voisins n'ont pas été représentés afin de faciliter la lecture. 
D'autre part la figure 4 montre que la droite correspondant à la classe d'âge 3 se trouve à l'intérieur du secteur délimité par les droites considérées comme confondues (entre les classes 2 et 4 ). 11 semble donc justifié de considérer comme confondues les droites représentant les âges supérieurs à 10 ans.

Nous avons de plus remarqué que la somme des carrés des écarts correspondant à la régression qui décrit le groupe formé par les 3 classes d'âge définies comme confondues par le test de comparaison, additionnées de la somme des carrés des écarts de l'équation séparée appliquée pour des âges du taillis compris entre 20 et 29 ans est supérieure à la somme des carrés des écarts obtenue en regroupant ces 4 classes d'âge.

Ceci nous amène à émettre des réserves sur l'utilisation du test de Barllut. Nous constatons en effet que même si 2 populations ne sont pas considérées comme comparables par ce test, ceci ne signifie nullement qu'elles sont significativement distinctes. SNEDECOR \& COCHRAN (1971) signalent d'ailleurs que ce test est particulièrement sensible à la non-normalité des populations, et notamment à l'aplatissement. En cas d'aplatissement positif ce test donne à tort beaucoup de verdicts d'hétérogénéité.

Nous admettrons donc qu'une seule équation est acceptable pour toutes les classes d'âge, y compris pour les 3 individus de la classe 1, qui ne s'éloignent pas du reste du nuage.

\subsection{Région forestière :}

(1) Sologne, (2) Orléanais, (3) Pays Fort. (4) Champagne berrichonne,

(5) Boischaut Nord

Nous pouvons appliquer une seule équation pour les 3 premières régions. En ce qui concerne les 2 dernières, il faut se garder de trop tirer de conclusions, les 2 régressions n'étant calculées que pour 5 et 4 individus. Nous n'attacherons donc pas de grande importance aux 2 équations séparées. Notons également sur la figure 5 que les 2 droites correspondantes sont très peu éloignées des 3 premières.

\subsection{Type de sol}

Le test de comparaison a permis de définir 2 grands groupes de sols :

- sols brun hydromorphe, brun non hydromorphe, lessivé non hydromorphe, sont comparables entre eux et confondus;

- sols lessivé hydromorphe, podzolique, très hydromorphe, ils sont également comparables et confondus.

Ils correspondent sensiblement pour le $1^{\text {rr }}$ groupe à un sol forestier favorable, et pour le $2^{\mathrm{e}}$ groupe à un sol forestier de qualité plus moyenne. Le podzol, très dégradé et le plus défavorable, n'est pas comparable aux autres types de sol (fig. 6).

Nous pouvons appliquer pour la variable «type de sol" le même raisonnement que précédemment (classe d'âge), en notant que les courbes extrêmes sont considérées comme confondues par les tests. 


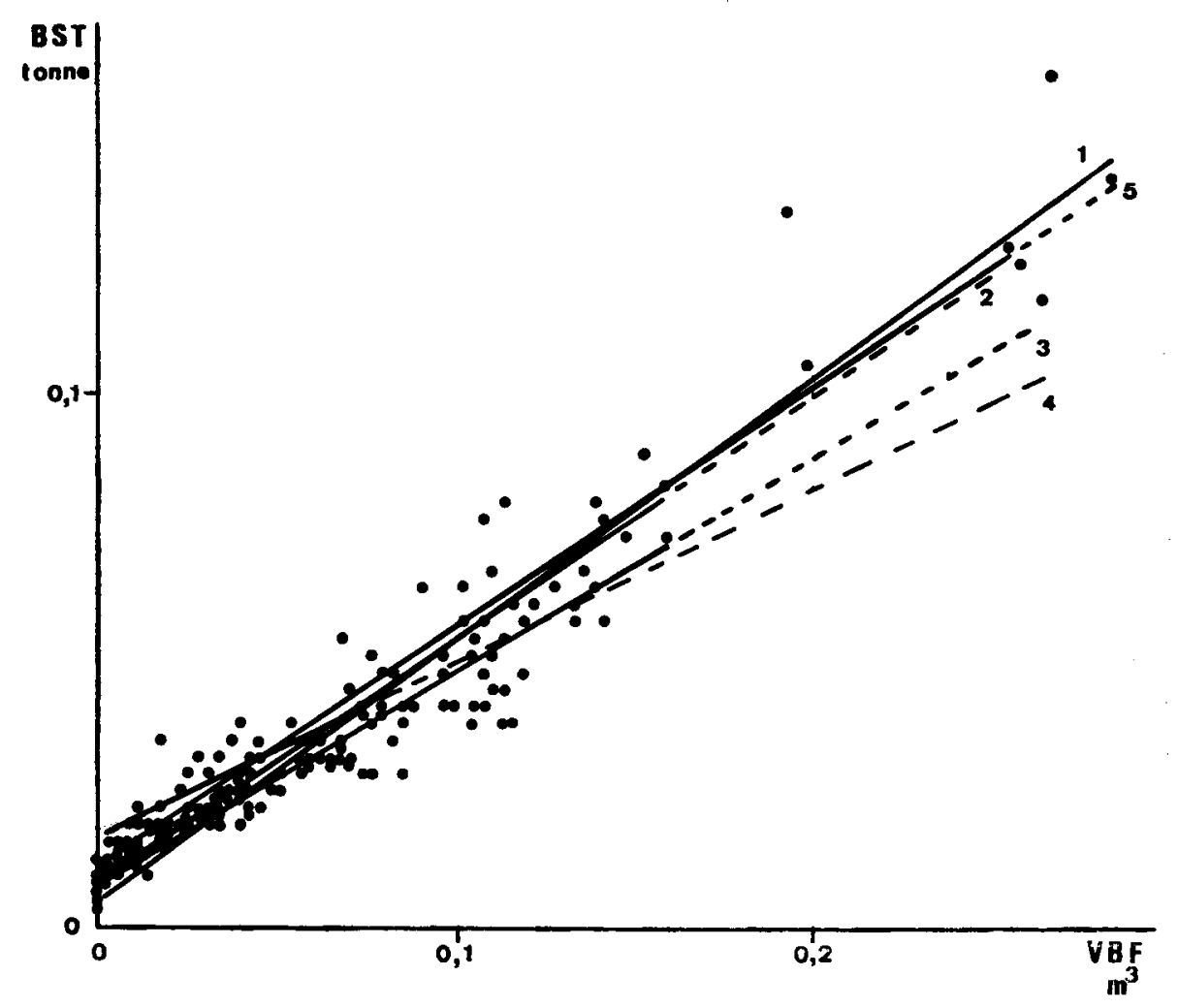

FIG. 5

Représentation graphique des droites de régression pour la variable « Régın forestière». Graph showing the 5 regression lines for the variable «Forest Region».

1. Sologne.

Sologne.

2. Orléanais. Orléanais.

3. Pays Fort. Pays Fort.

4. Champagne berrichonne. Champagne berrichonne.

5. Boischaut Nord. Boischaut Nord. 


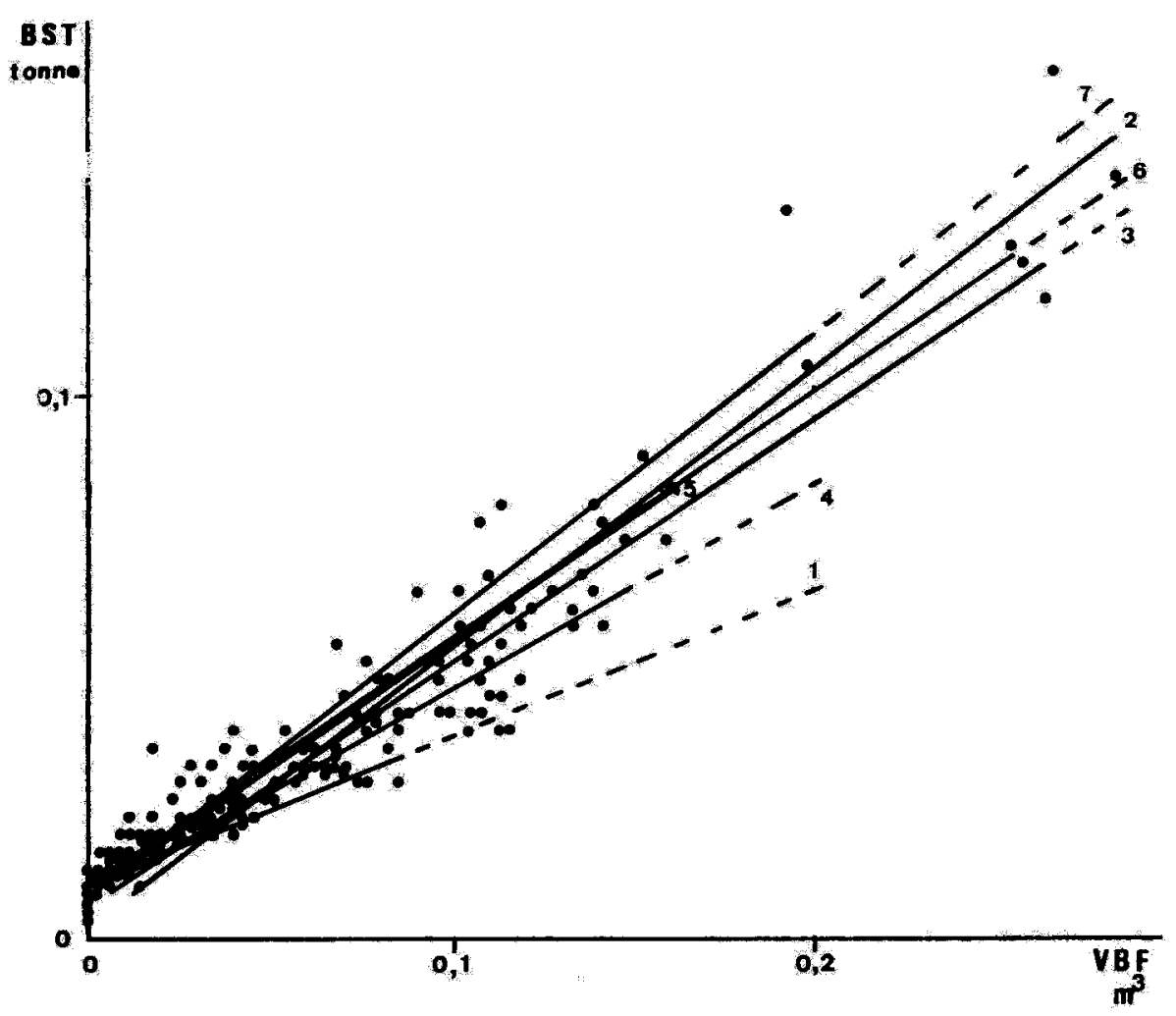

FIG. 6

Reprểsentation graphique des droites de rếgression poùr la variable «Type de sol s. Graph showing the 7 regression lines for the variable \& Soil type».

\section{Podzol. \\ Podsol.}

2. Podzolique.

Poilsolic.

3. Lessivé non hydromorphe.

Dry leached.

4. Lessivé hydromorphe.

Hydromorphic leached.

5. Brun non hydromorphe.

Dry brown,

6. Brun hydromorphe.

Hydromorphic brown.

7. Très hydromorphe.

Water-logged. 


\subsection{Calcul d'erreur}

Nous avons été amenés à discuter l'utilisation des tests statistiques. En effet, si la population des résidus de la régression suit une loi normale (fig. 7), par contre la distribution des valeurs de VBF et BST s'éloigne nettement de la normale (fig. 8 et 9) : ces 2 populations ont une asymétrie très marquée vers les grandes valeurs.

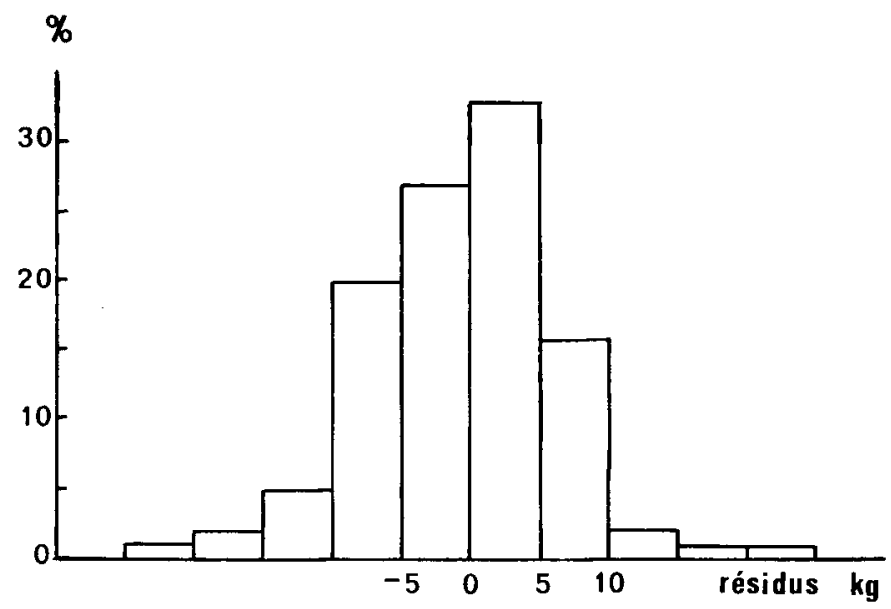

FIG. 7

Histogramme des résidus de la régression globale. Histogram of the residues of the global regression.

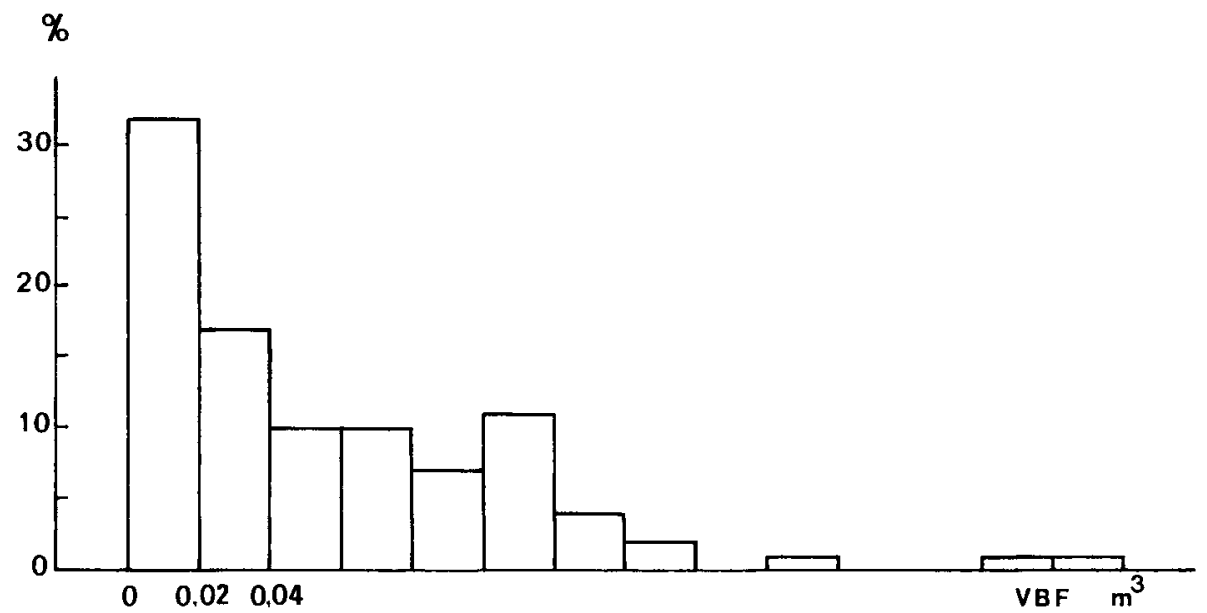

Fig. 8

Histogramme de la variable «Volume bois fort» pour la population globale $(V B F>0)$.

Histogram of the variable "Large timber volume» (VBF) for the global population $(V B F>0)$. 


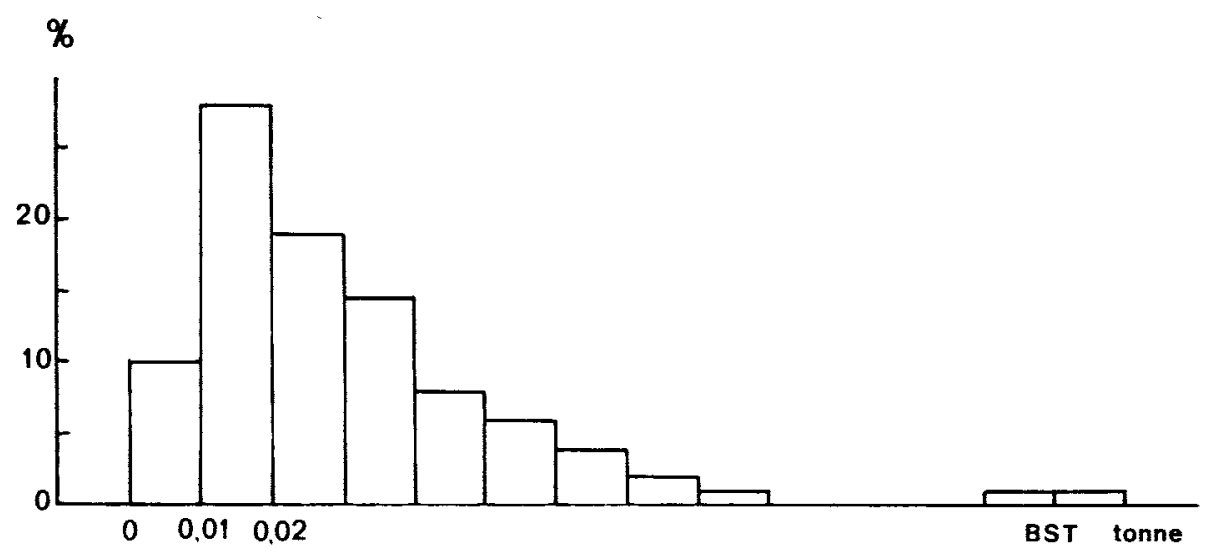

Fig. 9

Histogramme de la variable "Biomasse sèche totale» pour la population globale $(V B F>0)$.

Histogram of the variable "Total dry weight» (BST) for the global population $(V B F>0)$.

Ceci met en cause la fiabilité du test d'homogénéité des variances de BartLetT.

Pour une utilisation pratique de nos résultats il importe avant tout de considérer l'intérêt des régressions dans un but prédictif. Nous avons pour cela calculé l'erreur statistique rattachée à la régression :

Nous pouvons évaluer l'écart-type de l'estimation par la formuk:

$$
\begin{aligned}
& \mathrm{s}_{\hat{y}}=\mathrm{s}_{\mathrm{y} \cdot \mathrm{x}} \cdot \sqrt{1 / \mathrm{n}+\mathrm{x}^{2} / \Sigma \times 2} \quad \text { (SNEDICOR } \mathrm{k} \text { COCHRAN, 1971) } \\
& \text { avec } s_{y \cdot x}=\sqrt{\frac{\sum(y-\hat{y})^{2}}{n-2}}
\end{aligned}
$$

Pour l'équation de régression globali:

$s_{\hat{y}}=\sqrt{375000+0,000056(\overline{V B F}-\overline{V B \bar{F}})^{2}}$

Ceci donne pour l'individu moyen un écart-type de 1,8 p. 100, soit une erreur au risque 5 p. 100 de 3,5 p. $100(1,2 \mathrm{~kg}$ pour $B S T=34,2 \mathrm{~kg})$.

Ceci est très inférieur à l'erreur calculée par l'I.F.N. pour les estimations de volume. Nous pouvons donc accepter l'utilisation d'une équation unique, sachant que les valeurs sur lesquelles elle s'applique ont une incertitude supéricurc (ćcarttype de 2,5 p. 100 pour le Loiret). Ceci rejoint les conclusions de Drcourt (1973) 
qui constate qu'un tarif de cubage moyen apparaît plus précis qu'un tarif séparé par peuplement dans une région donnée.

\subsection{Etude des individus sans bois fort}

Dans l'étude des régressions nous avons laissé de côté les brins pour lesquels le diamètre à $1,30 \mathrm{~m}$ était inférieur à $7 \mathrm{~cm}$. Pour ceux-ci, $V B F=0$ mais $B S T>0$. Ils n'entrent pas en compte dans les données à l'I.F.N., mais peuvent représenter une quantité non négligeable dans certains peuplements, en particulier la classe d'âge 0 à 9 ans.

Afin d'évaluel la biomasse contenue dans ces individus nous avons calculé : $\Sigma_{0}$, la somme des BST des brins n'ayant pas de bois fort $(V B F=0)$ et $\Sigma_{1}$ la somme des BST des brins ayant du bois fort $(\mathrm{VBF}>0)$, sur l'échantillon représenté par les 96 parcelles étudiées. Nous avons effectué ce calcul pour chaque valeur de chacune des variables étudiées. Le résultat est donné au tableau 1.

Il importe de noter que ceci n'est qu'une approximation de la réalité sur le terrain, notre échantillon de 96 parcelles n'étant pas forcément représentatif. Cependant, en l'absence de données plus fiables, nous admettrons ces résultats provisoirement.

On peut noter que sur l'échantillon étudié la contribution des «petits brins" à la biomasse totale est en moyenne de 12 p. 100 . Ceci est très variable selon les conditions, en particulier l'âge : 78 p. 100 pour la classe d'âge 0 à 9 ans, et 0 p. 100 pour les âges supérieurs à 40 ans, mais également l'espèce : 30 p. 100 pour le charme et 8 p. 100 pour le robinier.

\subsection{Application aux données de l'I.F.N.}

Pour évaluer la biomasse sur pied des taillis nous avons appliqué les relations précédemment obtenues au volume de bois fort figurant dans les tableaux de l'I.F.N.

Cependant, les régressions ayant été calculées sur des brins individuels, il faut connaître le nombre d'arbres, en plus de leur volume. Ce réstultat n'est pas donné dans les tableaux généraux publiés par I'I.F.N. Il n'existe que dans les publications les plus récentes, et seulement pour certaines variables (espèce, région forestière). En ce qui concerne les données non publiées pour la région Centre, elles nous ont été aimablement communiquées par les services de l'I.F.N.

\subsection{Loiret}

Nous donnons dans le tableau 2 le résultat calculé pour le département du Loiret, essence par essence. Le calcul est effectué de la manière suivante :

1) Calcul de $\Sigma_{1}(\Sigma$ BST pour VBF $>0$ ). Les valeurs de $\Sigma$ VBF, et du nombre d'arbres $\mathrm{N}$ sont données dans le tome II de la publication de l'I.F.N. Nous avons appliqué la relation $\Sigma \mathrm{BST}=\mathrm{a} \Sigma \mathrm{VBF}+\mathrm{Nb}$ pour les 5 essences séparées, et pour la population globale. 


\section{TABLEAU 1}

Rapport du poids sec des brins n'ayant pas de bois fort au poids sec des brins ayant du bois fort.

Proportion of biomass for trees with no «large timber» to trees with «large timber».

\begin{tabular}{|c|c|c|c|}
\hline Variable & $\begin{array}{l}\therefore B S T(V B F=0) \\
\therefore \text { BST }(\mathrm{VBF}=0)\end{array}$ & $\begin{array}{l}\text { Nombre d'arbres } \\
\quad(\text { VBF }=0)\end{array}$ & $\begin{array}{c}\text { Nombre darbres } \\
(\text { VBF } \neq 0)\end{array}$ \\
\hline Global & 0,13 & 120 & 123 \\
\hline \multicolumn{4}{|l|}{ Peuplement } \\
\hline Taillis simpie $\ldots \ldots \ldots$. & 0,18 & 38 & 35 \\
\hline $\mathrm{TSF}<50$ p. $100 \ldots \ldots$ & 0,10 & 43 & 58 \\
\hline $\mathrm{TSF}>50$ p. $100 \ldots \ldots$ & 0,14 & 39 & 30 \\
\hline \multicolumn{4}{|l|}{ Essence } \\
\hline Chêne. & 0,08 & 17 & 39 \\
\hline Charme ........ & 0,43 & 43 & 13 \\
\hline Bouleau-tremble $\ldots \ldots \ldots$ & 0,10 & 25 & 28 \\
\hline Châtaignier .......... & 0,14 & 30 & 30 \\
\hline Robinier $\ldots \ldots$ & 0,09 & 5 & 13 \\
\hline \multicolumn{4}{|l|}{ Age } \\
\hline $0-9$ ans $\ldots$ & 3,59 & 29 & 1 \\
\hline $10-19$ ans $\ldots \ldots \ldots$ & 0,41 & 35 & 12 \\
\hline $20-29$ ans $\ldots \ldots \ldots \ldots$ & 0,18 & 39 & 45 \\
\hline $30-39$ ans $\ldots \ldots \ldots \ldots$ & 0,05 & 17 & 56 \\
\hline$>40$ ans $\ldots \ldots \ldots$ & 0,00 & 0 & 9 \\
\hline \multicolumn{4}{|l|}{ Région forestière } \\
\hline Sologne. & 0,10 & 46 & 70 \\
\hline Orléanais ......... & 0,23 & 50 & 34 \\
\hline Pays Fort $\ldots \ldots \ldots \ldots$ & 0,08 & 9 & 10 \\
\hline Champagne berrichonne . & 0,11 & 10 & 5 \\
\hline Boischaut Nord ....... & 0,06 & 5 & 4 \\
\hline \multicolumn{4}{|l|}{ Type de sol } \\
\hline Podzol .... & 0,47 & 9 & 6 \\
\hline Podzolique $\ldots \ldots \ldots \ldots$ & 0,06 & 7 & 13 \\
\hline Lessivé non hydromorphe & 0,09 & 26 & 40 \\
\hline Lessivé hydromorphe .... & 0,12 & 11 & 22 \\
\hline Brun non hydromorphe . . & 0,21 & 22 & 12 \\
\hline Brun hydromorphe ..... & 0,24 & 20 & 10 \\
\hline Hydromorphe $\ldots \ldots \ldots$ & 0,11 & 25 & 20 \\
\hline
\end{tabular}




\section{TABLEAU 2}

Volume de bois fort et biomasse sèche totale aérienne des brins de taillis par essence pour le Loiret. D'après les données IFN.

Volume of tres whose DBH over bark is over $7 \mathrm{~cm}$ and total above-ground dry weight of coppice by species for the Loiret.

\begin{tabular}{|c|c|c|c|c|}
\hline Essence & $\begin{array}{l}\text { VBF } \\
\left(\mathrm{m}^{3}\right)\end{array}$ & $\begin{array}{l}\text { Nombre } \\
\text { de tiges }\end{array}$ & $\begin{array}{c}\text { BST } \\
\text { (tonnes) }\end{array}$ & $\begin{array}{l}\text { Ecart-type } \\
\text { dû à la régression } \\
\text { (p. 100) }\end{array}$ \\
\hline Chêne ....... & 1937000 & 30540000 & 1524000 & 3,3 \\
\hline Charme et hêtre. & 772000 & 20709000 & 854000 & 5,8 \\
\hline Bouleau et tremble & 986000 & 15991000 & 688000 & 3,2 \\
\hline Châtaignier $\ldots \ldots$. & 272000 & 3998000 & 174000 & 3,7 \\
\hline Autres feuillus .......... & 887000 & 18521000 & 626000 & 2,9 \\
\hline Total LOIRET & 4854000 & 89759000 & 3641000 & 1,8 \\
\hline
\end{tabular}

2) La biomasse totale $\Sigma$ tient compte des brins sans bois fort et est donnée par :

$$
\Sigma=\Sigma_{1} \text { (calculé) } \times\left(1+\frac{\Sigma_{0}}{\Sigma_{1}} \text { (estimé) }\right)
$$

\subsection{Région Centre}

Les données non publiées dans les fascicules de l'l.F.N. nous ont élé communiquées par le service de l'I.F.N. Ceci nous a permis par le même calcul que précédemment de donner au tableau 3 la biomasse sèche totale aérienne sur pied des taillis, par département. Nous avons appliqué ici la seule équation globale, pour tous les départements de la région.

\section{TABleau 3}

Volume de bois fort et biomasse sèche totale aérienne des brins de taillis de la région Centre, par département. D'après les donnés IFN.

Volume of trees whose $D B H$ over bark is over $7 \mathrm{~cm}$ and total above-ground dry weight of coppice for the region Centre, by county.

\begin{tabular}{|c|c|c|c|c|}
\hline Département & $\begin{array}{c}\text { Superficic } \\
\text { (ha) }\end{array}$ & $\begin{array}{l}\mathrm{VBF} \\
\left(\mathrm{m}^{3}\right)\end{array}$ & $\begin{array}{l}\text { Ncmbre } \\
\text { de tiges }\end{array}$ & $\begin{array}{c}\text { BST } \\
\text { (tonnes) }\end{array}$ \\
\hline Cher ...... & 120760 & 4222000 & 88335000 & 3252000 \\
\hline Eure-ct-Loir & 54840 & 2722000 & 55404000 & 2084000 \\
\hline Indre $\ldots . .$. & 73150 & 2853000 & 66492000 & 2253000 \\
\hline Indre-et-Loire & 93750 & 5124000 & 97082000 & 3863000 \\
\hline Loir-et-Cher & 105500 & 2825000 & 72382000 & 2285000 \\
\hline Loiret . . . . . & 113360 & 4854000 & 89759000 & 3641000 \\
\hline Total RÉGION CENTRE . . & 561360 & 22601000 & 469455000 & 17378000 \\
\hline
\end{tabular}




\subsection{Discussion}

On peut remarquer une discordance dans le tableau 2 : en additionnant BST pour toutes les essences on obtient un total différent de celui donné dans le tableau. Celui-ci a été calculé directement par l'équation globale. Elle est plus précise (écart-type 1,8 p. 100 ) et la fiabilité de cette dernière quantité est meilleure que par l'application des 5 équations séparées. Ceci s'explique aisément par le fait que l'équation globale ait été calculée sur un nombre beatcoup plus grand d'individus que chacune des équations séparćes et par le fait que ces équations sont assez proches l'une de l'autre.

Il semble donc plus logique en pratique de n'appliquer qu'une seule formule, issue de l'équation globale.

II est intéressant de comparer les résultats obtenus ici aux calculs de BouchoN et al. (1981). Ceux-ci ont appliqué aux données de l'I.F.N. un simple coefficient multiplicateur, calculé d'après les données de Auclair \& Metayer (1980). Ils ont calculé le rapport moyen sur 3 peuplements de la biomasse totale à la biomasse «bois fort», qui était de 1.82. Après avoir appliqué ce coefficient au volume bois fort, ils ont multiplié ce résultat $\left(\mathrm{en} \mathrm{m}^{3}\right)$ par le coefficient 0,55 pour obtenir une biomasse sèche totale (en tonnes) :

et donc :

$$
\text { BST (tonnes) }=\operatorname{VBF}\left(\mathrm{m}^{3}\right) \times 1,82 \times 0,55
$$

$$
\text { BST (tonnes) }=\operatorname{VBF}\left(\mathrm{m}^{3}\right)
$$

$\mathrm{Ce} 1^{\mathrm{n}}$ résultat était calculé sur les seules données disponibles à l'époque. Nous pouvons effectuer un calcul similaire avec les données recueillies sur les 277 brins de taillis étudiés ici.

1) Une $1^{\text {re }}$ méthode consiste à calculer la moyenne sur les 277 brins de taillis :

- le rapport biomasse totale/biomasse bois fort $=1,56$,

- le rapport biomasse bois fort (grammes)/volume bois fort $\left(\mathrm{cm}^{3}\right)=0,53$.

On en déduit le coefficient multiplicateur moyen :

$$
\text { BST (tonnes) }=\mathrm{VBF}\left(\mathrm{m}^{3}\right) \times 0,82
$$

2) Une méthode plus rigourcuse consiste à tenir compte des équations de régression calculées ici :

- Pour les brins ayant du bois fort :

donc :

$$
\mathrm{BST}=\mathrm{a} \mathrm{VBF}+\mathrm{b}
$$

$$
\begin{aligned}
& \Sigma B S T=a \Sigma V B F+N b \\
& \Sigma B S T=\Sigma V B F \times(a+b \times N / \Sigma V B F)
\end{aligned}
$$

Nous pouvons calculer le volume bois fort moyen, sur la région Centre :

$$
\sum \mathrm{VBF} / \mathrm{N}=48143 \mathrm{~cm}^{*}
$$

Prenons l'équation globale :

$$
a=0,569 \text { et } b=7898
$$


On obtient :

$$
\Sigma \mathrm{BST}=\Sigma \mathrm{VBF} \times 0,733
$$

- En tenant compte des brins sans bois fort, on applique le coefficient multiplicateur 1,13 (tabl. 1):

$$
\Sigma \mathrm{BST}=\Sigma \mathrm{VBF} \times 0,83
$$

L'évaluation de Bouchon et al. (1981) était basée sur 3 parcelles particulières de taillis, dans lesquelles la proportion de bois fort était de 55 p. 100. En faisant la moyenne sur les 277 brins étudiés ici, nous obtenons 64 p. 100 . Cette différence s'explique aisément par un biais dans l'échantillonnage effectué précédemment.

Il est intéressant de noter que la méthode plus rigoureuse tenant compte des équations calculées ici donne un résultat très semblable à la première.

\section{Conclusion}

Grâce au travail de l'Inventaire forestier national, nous possédons des données sur le volume de la forêt française, selon les différentes régions, essences, âges, etc. Le présent travail propose une méthode permettant de convertir ces données en biomasse sèche totale aérienne. Si quelques-unes des variables étudiées ont une influence non négligeable sur les coefficients de la régression (en particulier l'âge : classe d'âge 0 à 9 ans), nous pouvons admettre en général que l'équation globale (1) BST $(\mathrm{g})=0,569$ VBF $\left(\mathrm{cm}^{3}\right)+7898$ est satisfaisante, avec un écart-type de 1,8 p. 100.

Cette équation n'est rigoureusement valable que sur la population échantillonnée. Nous pouvons cependant admettre de l'étendre à toute la région Centre, où les conditions moyennes sont relativement constantes.

En pratique, connaissant le volume bois fort VBF et le nombre d'arbres $\mathrm{N}$, on calcule dabord la biomasse sèche totale BST $_{1}$ pour les arbres ayant du bois fort avec l'équation (1), puis on applique le coefficient tenant compte des arbres sans bois fort pour connaître BST global (coefficient 1,13 pour la population globale).

Pour étendre ces résultats à toute la France, il faudrait poursuivre les échantillonnages sur tout le territoire. Il est par exemple très hasardeux d'utiliser cette équation sur les taillis méditerranéens, très différents par les espèces présentes, les sols et le climat.

D'autre part, il serait également intéressant d'obtenir des renseignements concernant la partie souterraine. Les évaluations parfois citées (15 p. 100) ne sont basées la plupart du temps que sur des résultats de la littérature sur des arbres de futaie.

Le présent travail remet en cause la $1^{\mathrm{r} r}$ évaluation de Bouchon et al. (1981). Un coefficient multiplicateur global de 0,83 tonne sèche par mètrc cube est proposé ici, avec toutes les réserves ci-dessus. 
Les données de Bouchon et al. (1981) peuvent être actualisées, grâce à des données plus récentes de l'I.F.N. aimablement communiquées par M. Rousseau :

- surface de taillis : 2,5 millions d'hectares,

- surface de taillis-sous-futaic : 4,25 millions d'hectares, soit au total : 6,750 millions d'hectares,

— volume total «bois fort» sur pied : 250 millions de $\mathrm{m}^{3}$,

— accroissement annuel moyen : 14 millions de $\mathrm{m}^{3}$,

— biomasse sèche totale aérienne sur pied : 185 millions de tonnes,

- accroissement annuel moyen : 11,8 millions de tonnes.

\section{Remerciements}

Nous tenons à remercier MM. Bertrand et Rousseau du Service de I'Inventaire forestier national pour nous avoir fourni très rapidement toutes les données qui nous ont été nécessaires, M. Formery et les techniciens du C.R.P.F. et M. Roussizal du S.R.A.F. pour l'aide qu'ils ont apportée à la prospection forestière, ainsi que tous les gestionnaires de l'Office National des Forêts et les propriétaires privés qui nous ont accueillis et nous ont facilité la tâche dans leurs forêts.

Nous remercions également $M$. Bouchon et les membres du "groupe des dendrométriciens $\gg$ pour leurs critiques et leur contribution à cette étude.

Ce travail a été effectué grâce à une participation financière du Commissariat à l'Energie solaire (contrat C.O.M.E.S. 70.75.102).

\section{Summary \\ A regional biomass survey method for coppice, using the National Forest Inventory data. Application to Central France}

The french National Forest Inventory gives data concerning «large timber» volume (VBF) of forest stands, in relation to various regions, species, ages, etc.

The present study gives a method to convert these figures into total above-ground dry biomass data (BST). A simple linear regression of the form BST $=a \mathrm{VBF}+\mathrm{b}$ gave good results : $\mathrm{r}^{2}=0,961$ for 142 individual trees sampled on 96 coppice and coppice with standard stands distributed throughout the french «Région Centre» (fig. 1). A few of the studied variables have some effect on the regression coefficients (for instance age-class 0-9 yrs containing very small stems). The global equation is neverthcless very satisfactory with a standard deviation of $1.8 \mathrm{p} .100$ for the mean.

A study of the small trees with no "large timber» $(\varnothing>7 \mathrm{~cm})$ showed that these only contain $12 \mathrm{p} .100$ of the total biomass in the sampled stands. Using the abovementioned regression equation and taking into account the small trees allowed us to give a global estimation of above-ground standing biomass in the region Centre. An extrapolation to other regions is suggested, but should be based upon a more extensive sampling to be sufficiently valid.

Reçu le 22 septembre 1982.

Accepté le 10 décenbre 1983. 


\section{Références bibliographiques}

Alemdag 1.S., 1982. Methods of estimating forest biomass from stand volumes : A case study with Ontario Jack Pine. Pulp Paper Canada, 83, 41-43.

Auclair D., Metayer S., 1980. Méthodologie de l'évaluation de la biomasse aćrienne sur pied et de la production en biomasse des taillis. Acta Ecologica, Ecol. Appl., 1, 357-377.

BiGE M.C., 1982. Evaluation de la biomasse et de la productivité des taillis de la région Centre. Méthodologie et premiers résultats. I.N.R.A., Station de recherches sur la forêt et l'environnement, doc. $82 / 38,52 \mathrm{p}$. + annexes.

Bouchon J., Ottorini J.M., PARdé J., 1981. Contribution à une meilleure connaissance des potentialités ligneuses totales en France à partir des données de l'Inventaire forestier national. Rapport C.E.E., projet F. 203 (470.78.7, E.S.F.), 33 p.

Decourt N., 1973. Remarque sur deux modes de cubage des peuplements : mesures directes ou tarif moyen. Rev. For. Fr., 25, 308-311.

INVENTAIRE FORESTIER NATIONAL, 1972. But et méthodes de l'Inventaire forestier national. Ministère de l'Agriculture, Service des Forêts, 38 p.

KozaK A., 1972. A simple method to test parallelism and coincidence for curvilinear, multiple linear and multiple curvilinear regressions. In : I.U.F.R.O., $3^{*}$ Conférence du Groupe consultatif des Statisticiens forestiers. I.N.R.A., Paris, p. 133-145.

MADGWICK H.A.I., 1976. Mensuration of forest biomass. In : Young H.E., Ed., Oslo biomass studies. College of life sciences and agriculture, University of Maine at Orono, U.S.A., p. 11-27.

PARDÉ J., 1961. Dendrométrie. E.N.G.R.E.F., Nancy, 350 p.

PARDÉ J., 1980 a. Forest Biomass. For. Abstr., 41, 343-362.

Pardé J., 1980 b. Problèmes de biomasses forestières énergétiques. C.E.N.E.C.A. Colloquc international «Agriculture et Energie», Paris, 27-2-1980, 7 p.

Ranger J., Nys C., Ranger D., 1981. Etude comparative de deux écosystèmes forestiers feuillus et résineux des Ardennes primaires françaises. I : Biomasse aérienne du taillis-sous-futaic. Ann. Sci. For., 38, 259-281.

Snedecor G.W. et Cochran W.G., 1971. Méthodes statistiques. A.C.T.A., Paris, 649 p.

YOUNG H.E., 1976. Oslo biomass studies. College of life sciences and agriculture, University of Maine at Orono, U.S.A., 302 p.

Young H.E., 1981. Kyoto biomass studies. College of life sciences and agriculture, University of Maine at Orono, U.S.A., 171 p. 